\title{
Cellular Assays with Multiparametric Bioelectronic Sensor Chips
}

\author{
Joachim Wiest, Martin Brischwein, Johann Ressler, Angela M. Otto, Helmut Grothe, and \\ Bernhard Wolf*
}

\begin{abstract}
During recent years, sensor chip-based systems have been developed which monitor functional changes of living cells. Key elements of such cellular assays are silicon or glass chips with integrated sensors for physicochemical parameters, i.e. $\mathrm{pH}, \mathrm{pO}_{2}$, electric impedance and temperature. Analysis of these primary parameters yields information about cell metabolism, cell growth and adhesion, and cell morphological changes. The operation principles of individual sensor types are described together with examples of experimental results.
\end{abstract}

Keywords: Cell-based assay · Cell morphology · Cellular respiration · Extracellular acidification · Sensor chip

\section{Introduction}

Cell-based assays are essential tools in biomedical research [1], particularly in pharmaceutical drug screening [2] and medical diagnostics [3-5]. Generally, the reaction of cells or tissue upon addition of active agents is studied by endpoint analysis of a single parameter, for example by labeling with fluorescent protein analogs [6] or synthetic dyes [7]. However, no appropriate fluorescence techniques are presently available which can monitor changes of cell morphology and metabolic rates at the same time in living cells.

To obtain a multiparametric and dynamic analysis of cellular behavior, silicon and glass sensor chips were constructed which integrate ion sensitive field effect transistors (ISFETs) [8][9] to measure extracellular acidification, a planar amperometric electrode structure to measure cellular oxygen exchange [10], an interdigitated electrode structure (IDES) [11] to measure the density of adherent cells and changes

${ }^{*}$ Correspondence: Prof. Dr. rer. nat B. Wolf Technische Universität München

Heinz Nixdorf - Lehrstuhl für Medizinische Elektronik Arcisstrasse 21

D-80333 München, Germany

Tel.: +49 8928922947

Fax: +498928922950

E-Mail: wolf@tum.de

www.Ime.ei.tum.de in cell adhesion, and a diode or Pt1000 for temperature measurement.

Since data were acquired continuously for up to several days from cells cultured on the chips, a cell culture maintenance system was required. Therefore the temperature was maintained at $37^{\circ} \mathrm{C}$. A fluidic system was connected to the culture set-up, which allowed for the regular exchange of media, addition of drug solutions, and the adjustment of a small microvolume of culture medium on the chips. A defined microvolume is necessary to achieve a sufficiently high ratio of cell number to medium volume for sensitive cell metabolic recordings [12]. Some basic design considerations for microfluidic systems to meet microenvironmental requirements of cells have been summarized by Walker et al. [13].

It must be emphasized that this system was not intended for single cell analysis. The signals obtained were generally from cell populations growing directly on the sensors at the chip surface. Besides, the interest was not primarily directed to the determination of absolute metabolic rates or distinct cell morphological conditions, but to relative changes of these parameters in the course of an experiment

Multiparametric measurements showing the response of living cells to addition of different drugs are presented and the systems and sensors used are introduced.

\section{Materials and Methods}

The LS174T adenocarcinoma colorectal cell line (ATCC (CL 1-188)) was used as a model, since it has been well characterized in previous work in the group's laboratory. These cells were maintained in Dulbecco's Modified Eagle's Medium (Sigma, Munich, Germany) supplemented with $10 \%$ FCS (Seromed, Biochrom, Berlin, Germany).

For use in cellular assays the chips were sterilized by autoclaving. The cell-culturing procedure involved seeding a cell suspension directly on the chip surface $\left(10^{4}-10^{5}\right.$ cells) and incubation for $48 \mathrm{~h}$ in a humidified atmosphere with $5 \% \mathrm{CO}_{2}$. Fig. 1 shows adherent cells cultivated on a chip surface. Before inserting the chip into the test apparatus, the tubing was disinfected with $70 \%$ ethanol and subsequently rinsed with sterile deionized water and culture medium.

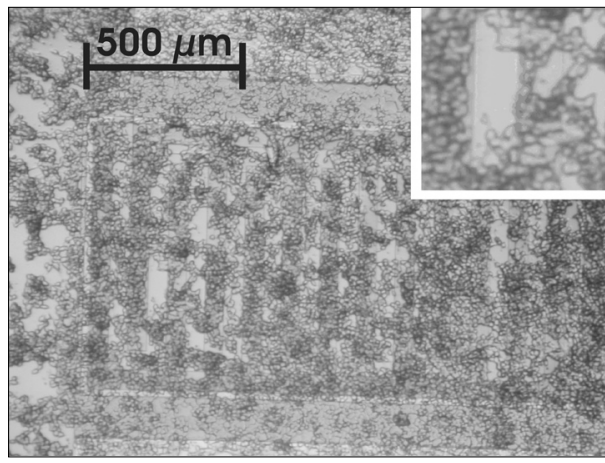

Fig. 1. Photograph of a LS $174 \mathrm{~T}$ cell culture on an IDES. The cells were seeded on the chip $48 \mathrm{~h}$ before. Insert with higher magnification in the corner

Two versions of sensor chips have been fabricated. 1) A silicon sensor chip (Fig. 2) with three different sensor types for multiparametric cell monitoring in nMOS technology. This silicon sensor chip was 


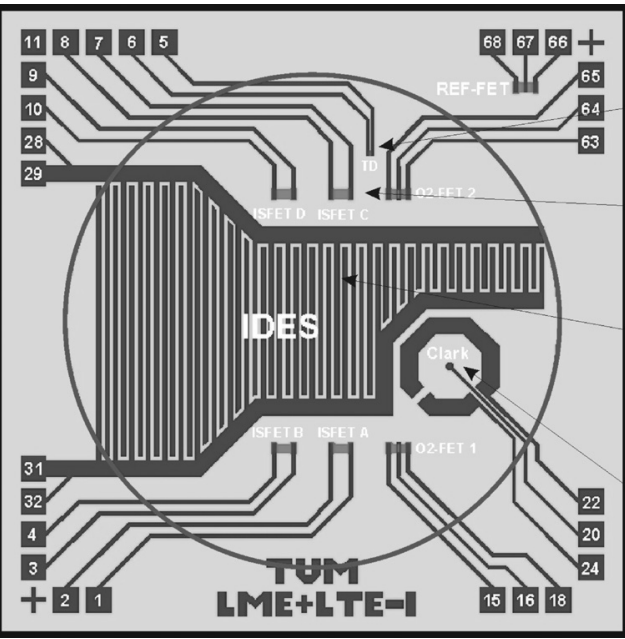

temperature

diode

PH-ISFETS

interdigital

electrode

structure

amperometric

oxygen sensor
Fig. 2. Silicon chip layout for bioelectronic assays. The outer dimensions are $7.5 \times 7.5 \mathrm{~mm}$. Four pH-ISFETs, one interdigitated electrode structure, one amperometric sensor structure and a temperature diode are integrated. bonded on a PLCC68 format standard printed circuit board and encapsulated by injection molding to create a culture vessel of approximately $9.5 \mathrm{~mm}$ in diameter (Fig. 3). 2) A glass sensor chip is shown in Fig. 4. It contains a silicon chip insert with ISFETs, IDES and amperometric electrode structures (both from platinum). These glass chips are housed in a chamber device providing electric contacts and fluid channels (Fig. 5). The cell culture area is $12 \mathrm{~mm}$ in diameter.

Acidification of the culture medium produced by cellular metabolism was measured with an ion-sensitive field effect transistor (ISFET) with gate regions of about $10 \mu \mathrm{m} \times 100 \mu \mathrm{m}(\mathrm{L} \times \mathrm{W})$. In such transistors, a low current passing through a semiconductor channel between two electrodes ('source and drain') was controlled by the voltage of a third, non-metallic 'gate-area' on top of this channel. The gate was covered with an insulating material which binds selectively protons, resulting in a $\mathrm{pH}$-dependent surface potential. With silicon nitride as gate isolator material, a pH-sensitivity between 40 and $55 \mathrm{mV} / \mathrm{pH}$ was obtained. Due to the $\mathrm{pH}$-sensitivity of the structures, the rate of extracellular acidification of cells growing directly on the sensors could be detected [14][15].

Oxygen consumption resulting from cellular respiration was measured with an amperometric oxygen sensor. This is a three electrode system where a constant potential of $-600 \mathrm{mV}$ is applied between a reference and a working electrode. Additionally, an auxiliary electrode was used to keep the

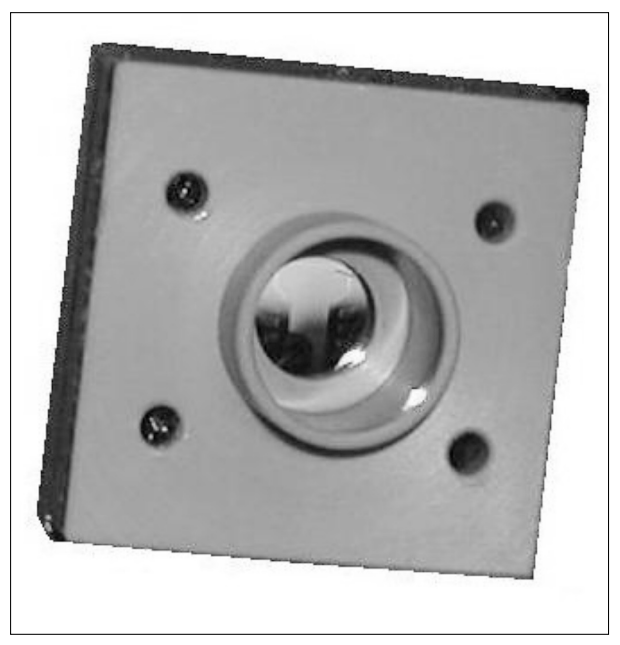

Fig. 3. Bonded and encapsulated silicon chip. The chip is bonded to a printed wire board (24 $\times 24 \mathrm{~mm}$ ) and packaged to form a free circular opening (diameter: $6 \mathrm{~mm}$ ) for cell culturing.

reference electrode without current (potentiostatic method [16]). The resulting current essentially depends on the concentration of the oxygen in the electrolyte. All three electrodes were made of palladium (silicon chip) or platinum (glass chip).The relative surface areas of working-, reference-, and auxiliary electrodes are about 1:1000:3000 (the area of the working electrode is about $700 \mu \mathrm{m}^{2}$ ). The relatively small area of the work electrode area ensured that all measured changes in the electrochemical current were due to changes at this electrode and that oxygen self-consumption and flow dependence were minimized. The structure was not covered by an oxygen diffusion membrane. Instead, the analyte solution itself was used as the electrolyte. To avoid interferences between the different sensor

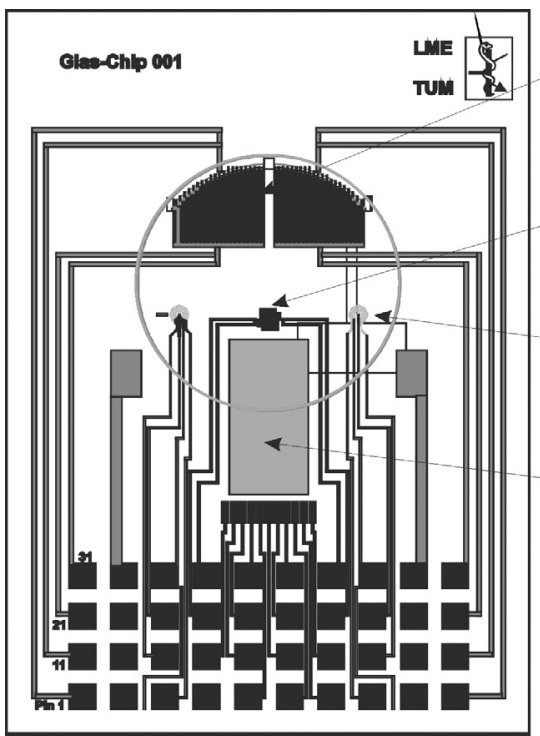

interdigital

electrode

structures

$\mathrm{P}+1000$

temperature

sensor

amperometric

oxygen sensors

pH Sensors/

area for silicon

insert
Fig. 4. Glass chip layout. The outer dimensions are $24.0 \times 33.8 \mathrm{~mm}$. Two interdigitated electrode structures and two amperometric electrode structures are integrated. For $\mathrm{pH}-$ measurement, a 3.5 $\times 7.0 \mathrm{~mm}$ silicon chip can be inserted to form a glass-silicon hybrid. Electric connections are made with needle contacts.

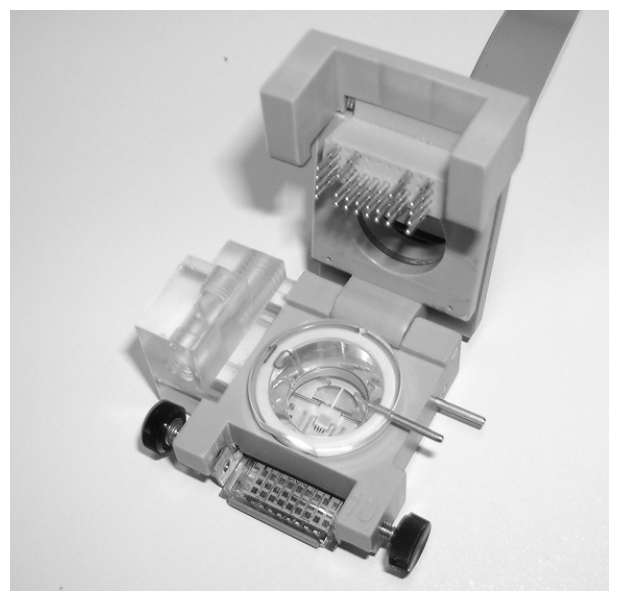

Fig. 5. Glass chip device: The chamber accomodates a single glass sensor chip. The sensors are pin-contacted, preamplification of weak electric sensor signals (e.g. oxygen sensor) is possible directly behind the contacting socket. Electronic boards and PC for sensor control and data acquisition are external. The height of the cell culture chamber is adjusted to $0.5 \mathrm{~mm}$. 
types, the amperometric sensor was galvanically insulated.

Changes in cell morphology were monitored with an interdigitated electrode structure (IDES) with an electrode width and electrode distance of $50 \mu \mathrm{m}$. A low-amplitude alternating current in a frequency range of about $10-20 \mathrm{kHz}$ was applied to the electrode structure. Due to the insulating properties of cell membranes in this frequency range, the measured impedance values increased upon cell adherence. Thus, changes in impedance reflect the process of cell spreading/cell adhesion and subtle rearrangements of the cytoskeleton, linked to cell-cell and cell-matrix junctions.

To measure the temperature with the silicon chip, a PN diode was used. With a forward-biased current applied at a diode, the resulting voltage is temperature dependent. To measure the temperature with the glass chip, a Pt1000 temperature dependent platinum resistor was used.

The periodic operation of the fluid perfusion system (typically 7 min flow-off followed by 3 min flow-on with a rate of 100 $\mu \mathrm{l} / \mathrm{min}$ ) shaped a periodic oscillation of $\mathrm{pH}-$

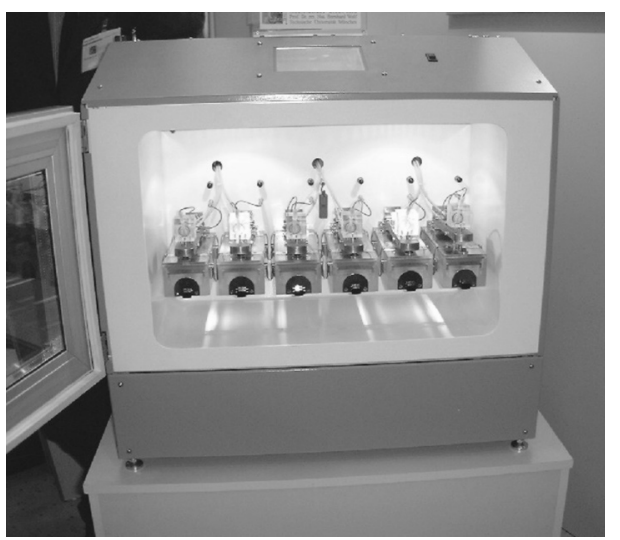

Fig. 6. Six-channel cell analyzer with integrated incubator and automated liquid handling system. An integrated PC provides system control (touchscreen user interface) and data storage for bioelectronical based assays.

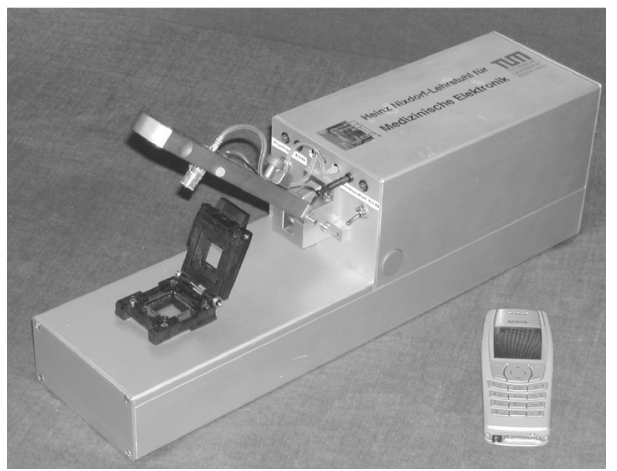

Fig. 7. Bioelectronic cell chip module using cellular assays for mobile applications with wireless data transfer. Data acquisition is performed by a $\mathrm{C}++$ application on an external laptop. and $\mathrm{pO}_{2}$ sensor signals. During the flow-off intervals, cells growing on the sensors affected the surrounding cell culture medium directly by extrusion of acidic metabolic products and by consuming dissolved oxygen. To evaluate the ISFET raw data, the slope of the graph (voltage vs. time) during the flow-off interval was calculated by linear regression. The resulting value was expressed as 'dU/dt'. Raw data of the amperometric sensor (current vs. time) were calculated in a similar way, except that only the first three minutes of the flow-off interval were considered and the resulting slope was corrected by the sensitivity of the sensor, which often changed during long term measurements. The resulting value was expressed as ' $\mathrm{dI} / \mathrm{dt}$ '. A measure of sensitivity was obtained from the sensor signal during the flow-on intervals, when fresh culture medium saturated with oxygen flowed into the microchamber. Thus, every flow cycle resulted in exactly one value of $\mathrm{dU} / \mathrm{dt}$ and $\mathrm{dI} / \mathrm{dt}$. Here, absolute values of $\mathrm{pH}$ and $\mathrm{pO}_{2}$ were not of interest. Therefore, the calculation of cellular metabolic rates was based on the determination of relative changes during short time intervals, where long term signal drift is negligible. In order to obtain baseline signals (sensor readings without viable cells), the non-ionic detergent Triton X-100 (0.1\%) was added at the end of the experiments. Triton disrupts cellular membranes and causes the immediate cessation of cell metabolism.

Three versions of cellular analysis systems have been realized: 1) a six-channel device (Fig. 6) as a first step towards parallel processing and automation of experiments, 2) a single channel version (Fig. 7) with emphasis on mobility, wireless data

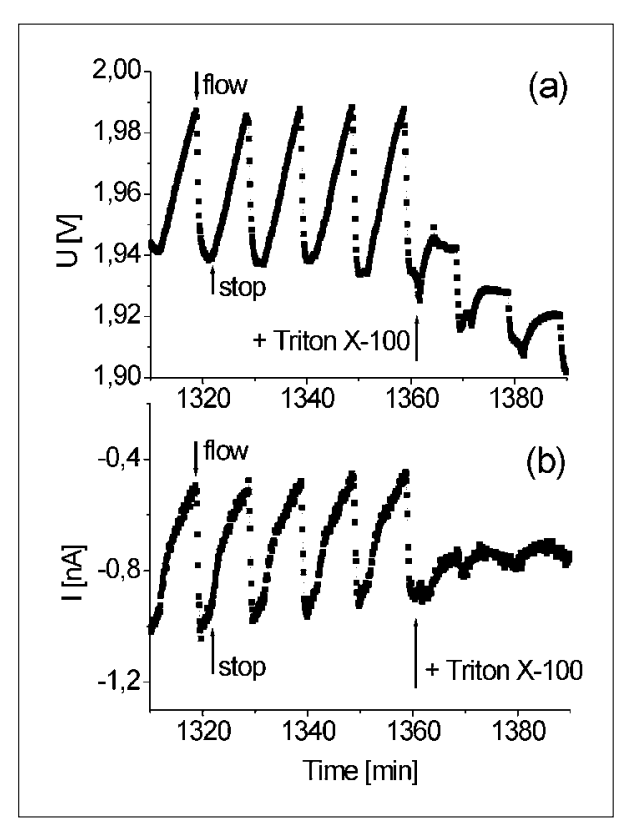

Fig. 8. Glass chip sensor raw data with Triton X100 lysis of cells. The periodic course due to cell metabolic activity and the operation of the fluid system is clearly visible. transfer and miniaturization, and 3) a device which can be placed on a microscope stage (Fig. 5) . Devices 1 and 2 are usually operated with silicon chips, while device 3 is equipped with glass chips.

\section{Results}

This sensor chip system has been used to study the metabolism of various cell types, such as continuous cell lines, human blood cells, tissue probes, bacteria, yeast and green algae [17] in different research projects.

As an example, the raw data of $\mathrm{pH}$ ISFETs and planar amperometric oxygen sensors with LS $174 \mathrm{~T}$ cells are shown in Fig. 8. To demonstrate that changes in different cell physiological parameters can be monitored in parallel, a drug with a well-characterized effect on these cells was added: Chloroacetaldehyde (CAA) is known to be a highly reactive, alkylating metabolite of the anticancer drugs ifosfamide and cyclophosphamide [18]. Addition of CAA at $50 \mu \mathrm{M}$ to LS $174 \mathrm{~T}$ cells in the sensor chip system leads to a rapid decrease in the rate of oxygen consumption (Fig. 9a). The rate of extracellular acidification declines as well, however, with a delay of about $2 \mathrm{~h}$ (Fig. 9b). These results have been reproduced by repeating the same experiment on different chips on different days. Changing impedance values indicate a gradual loss of cell adhesion to the chip substratum (Fig. 9c).

\section{Discussion and Outlook}

Dynamic monitoring of the effects of active agents on living cells is possible with multiparametric sensor systems based on silicon or glass chips. Sensor-based cell assays are non-invasive and do not need any optical fluorescence labels. To obtain information about cellular metabolism, a liquid handling system is operated in an interval mode and the kinetics of primary data of $\mathrm{pH}$ - and oxygen sensors are evaluated. The measurement of absolute values of $\mathrm{pH}$ and $\mathrm{pO}_{2}$ was not pursued. Only relative changes in the course of an experiment were analyzed and all interpretations were referenced to a baseline which was typically defined by the fresh cell culture medium properties at the end of one flow-on cycle.

From an economic point of view, the use of low-price technologies such as chips on glass or plastic could be superior to silicon. However, in order to make use of these economic advantages, the hybrid integration of small silicon chips with ISFETs into glass has to be replaced by a more convenient technology. Currently the suitability of alternative $\mathrm{pH}$ sensors based 


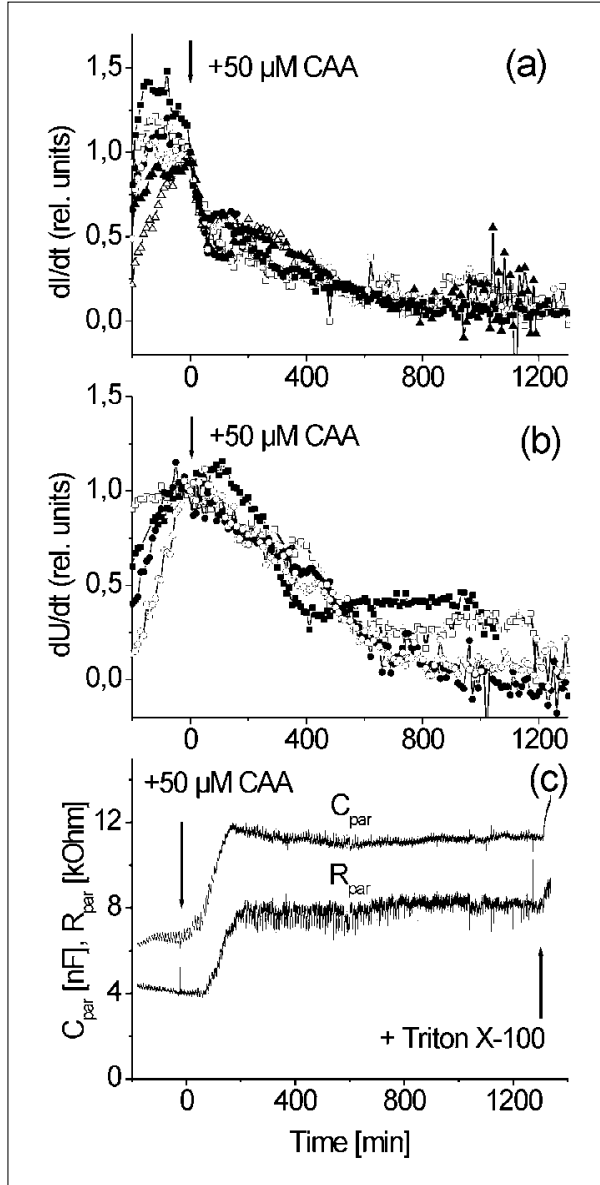

Fig. 9. Example for typical data pattern: Extracellular acidification (a), cellular oxygen consumption (b) and electric impedance (c) upon treatment of cells with $50 \mu \mathrm{M} \mathrm{CAA}$; Four graphs of extracellular acidification (dU/dt) and six graphs of oxygen consumption $(\mathrm{dl} / \mathrm{dt}$ ) are plotted in the same diagram to give an impression of reproducibility. The absolute values of dU/ $\mathrm{dt}$ and $\mathrm{dl} / \mathrm{dt}$ have been normalized for better comparison of the graphs. Impedance data of a single experiment are represented as the two components Cpar and Rpar.

on various metal oxides is being evaluated. Metal oxide sensors can be structured on glass with thin film or thick film process steps. Another feature important for many applications is a high throughput of experiments. Since in the pharmaceutical industry automated screening facilities use stacks of microtiter plates, an adequate array density for sensor-supported cell assays is imperative. Consequently, the development of multiwell plates equipped with electronic sensor chips is in progress. The bottom of culture wells has been replaced by chips of glass or polymeric substrate with integrated microsensors. This technology has been applied to a 24-well plate, which is compatible with existing microtiter plate equipment (Fig. 10). Engineering issues connected to the parallelization of sensor chips include novel ways for chip packaging and electric connections, as well as electronic sensor control and automated liquid handling.

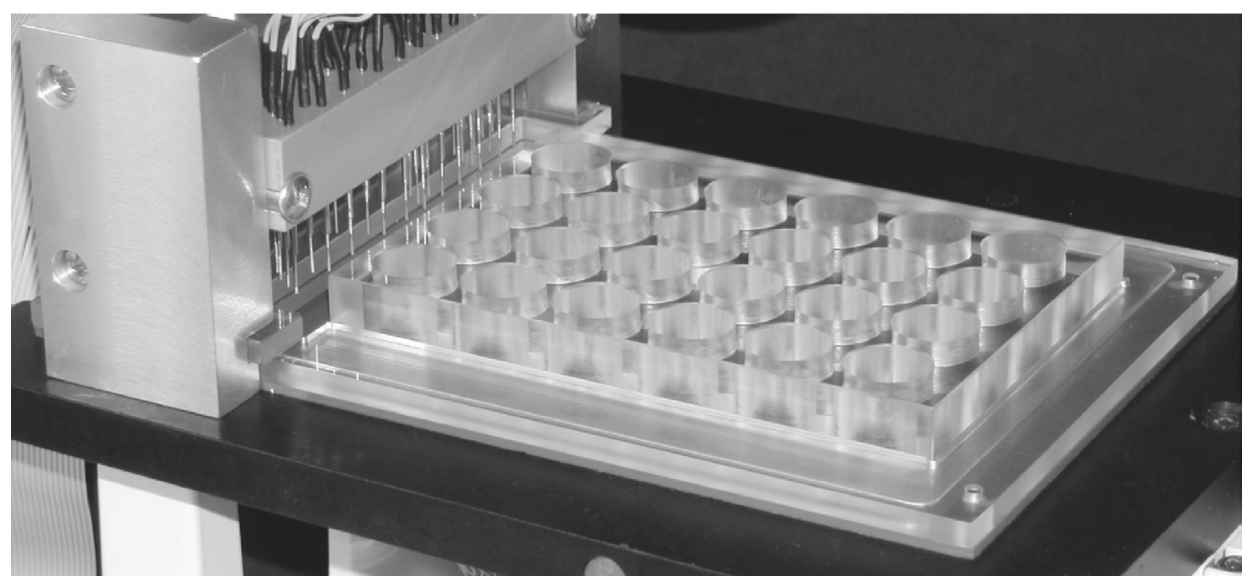

Fig. 10. Experimental setup for a 24-well plate. The glass bottom of the cell culture plate which allows microscopy is equipped with an array of 24 sensor units. Each unit has sensors for impedance, $\mathrm{pH}$ and $\mathrm{pO}_{2}$.

Further work is in progress to establish the long-term stability of the sensors, which is of particular importance for measurement with proliferating cells.

\section{Received: February 8, 2005}

[1] H. Andersson, A. van den Berg, 'Microfluidic devices for cellomics: a review', Sensors and Actuators 2003, B92, 315-25.

[2] I.A. Hemmilä, P. Hurskainen, 'Novel detection strategies for drug discovery', Drug Discovery Today 2002, 15, 150-156.

[3] E. Brown, M. Markman, 'Tumor chemosensitivity and chemoresistance assays', Cancer 1996, 77, 1020-1025.

[4] T. Henning, M. Brischwein, W. Baumann, R. Ehret, I. Freund, R. Kammerer, M. Lehmann, A. Schwinde, B. Wolf, 'Approach to a multiparametric sensor-chip-based tumor chemosensitivity assay', Anti-Cancer Drugs 2001, 12, 21-32.

[5] S. Ekelund, P. Nygren, R. Larsson, 'Microphysiometry: new technology for evaluation of anticancer drug activity in human tumor cells in vitro', Anti-Cancer Drugs 1998, 9, 531-538.

[6] A. Chiesa, E. Rapizzi, V. Tosello, P. Pinton, M. de Virgilio, K.E. Fogarty, R. Rizzuto, 'Recombinant aequorin and green fluorescent protein as valuable tools in the study of cell signalling', Biochem. J. 2001, 355, 1-12.

[7] E. Sullivan, E.M. Tucker, I.L. Dale, 'Measurement of $[\mathrm{Ca} 2+]$ Using the Fluorometric Imaging Plate Reader (FLIPR)', Methods in Molecular Biology 1999, 114, 125-134.

[8] P. Bergveld, 'The operation of an ISFET as an electronic device', Sensors and Actuators 1981, 1, 17-29.

[9] L.J. Bousse, 'The chemical sensitivity of electrolyte/insulator/silicon structures Fundamentals of ISFET operation', 1982.

[10] J. Wiest, M. Brischwein, H. Grothe, A.M. Otto, B. Wolf, 'Planar microsensors for measurement of cellular respiration, TEST Proceedings, 2005, (accepted for publication).

[11] R. Ehret, W.H. Baumann, M. Brischwein, A. Schwinde, K. Stegbauer, B. Wolf, 'Monitoring of cellular behaviour by impedance measurements on interdigitated electrode structures', Biosensors \& Bioelectronics 1997, 12, 29-41.

[12] J.C. Owicki, L.J. Bousse, D.G. Hafeman, G.L. Kirk, J.D. Olson, H.G. Wada, J.W Parce, 'The light-addressable potentiometric sensor: principles and biological applications', Annual Reviews of Biophysics and Biomolecular Structures 1994, 23 , 87-113.

[13] G.M. Walker, H.C. Zeringue, D.J. Beebe, 'Microenvironment design considerations for cellular scale studies', Lab on a Chip Miniaturisation for Chemistry, Biology \& Bioengineering 2004, 4, 91-97.

[14] M. Lehmann, A. Schwinde, R. Ehret, M. Brischwein, B. Wolf, W.H. Baumann, 'Microelectronic sensor system for microphysiological application on living cells', Sensors and Actuators 1999, B55, 77-89.

[15] S. Martinoia, N. Rosso, M. Grattarola, L. Lorenzelli, B. Margesin, M. Zen, 'Development of ISFET array-based microsystems for bioelectrochemical measurements of cell populations', Biosensors \& Bioelectronics 2001, 16, 1043-1050.

[16] A.J. Bard, L.R. Faulkner, 'Electrochemical Methods, Fundamentals and Applications', 2 ed., John Wiley \& Sons, Inc., 1969.

[17] M. Brischwein, H. Grothe, A.M. Otto C. Stepper, T. Weyh, B. Wolf, 'Ultrathin Electrochemical Chemo- and Biosensors', Ed. V.M. Mirsky, 2004, 159-180.

[18] C. Sood, P.J. O'Brien, 'Molecular mechanisms of chloroacetaldehyde-induced cytotoxicity in isolated rat hepatocytes', Biochemical Pharmacology 1993, 46, 1621-1626. 